\title{
Uterine artery blood flow and microvessel density by vaginal color Doppler ultrasonography in embryo implantation failure
}

\author{
ZHEN LI $^{1}$, XINGLING WANG ${ }^{1}$, YICHUN GUAN ${ }^{1}$, XIAONA YU $^{1}$, JING LIU ${ }^{1}$ and ZHAN ZHANG ${ }^{2}$ \\ ${ }^{1}$ Reproductive Center, The Third Affiliated Hospital of Zhengzhou University, Zhengzhou, \\ Henan 450052; ${ }^{2}$ Shangqiu Medical College, Shangqiu, Henan 476100, P.R. China
}

Received April 18, 2017; Accepted August 1, 2017

DOI: $10.3892 / \mathrm{etm} .2017 .5114$

\begin{abstract}
The purpose of this study was to investigate the clinical application of uterine artery blood flow parameters by vaginal color Doppler ultrasonography in patients with repeated embryo implantation failure and to examine the correlation between uterine artery blood flow parameters and microvessel density (MVD). We monitored the outcomes of in vitro fertilization and embryo transfer in eighty patients. We divided the patients into two groups: Pregnancy (successful pregnancy, $\mathrm{n}=50$ ) and repeated implantation failure (more than two failures of in vitro fertilization-embryo transfer, $\mathrm{n}=30$ ). The patients were subjected to vaginal color Doppler ultrasonography to measure endometrial thickness, peak systolic velocity/end diastolic velocity (S/D), pulsatility index (PI), resistance index (RI), and other uterine artery blood flow parameters. We found no significant differences in endometrial thickness (human chorionic gonadotropin endometrial thickness) and uterine artery blood flow parameters (S/D, PI) between the two groups. The RI value of endometrial blood flow was significantly higher in the repeated implantation failure group than that in pregnancy group. The endometrium MVD in the repeated implantation failure group was significantly lower than that of the pregnancy group. Spearman correlation analysis showed that $S / D$ value of uterine artery blood flow in repeated implantation failure group was not correlated with MVD or uterine artery blood flow PI, but was positively correlated with RI. Endometrium MVD in patients with repeated implantation failure was significantly lower than that in patients with successful pregnancy during the implantation window. We also found a significant difference in uterine artery blood flow RI between the two groups. The uterine artery blood flow RI in patients with repeated implantation failure was positively correlated with MVD, which has clinical significance in predicting the outcome of embryo transfer.
\end{abstract}

Correspondence to: Dr Zhan Zhang, Shangqiu Medical College, 7 Kangfuqian Street, Shangqiu, Henan 476100, P.R. China

E-mail: drzhanzhang1@hotmail.com

Key words: repeated implantation failure, uterine artery blood flow, vaginal color Doppler ultrasonography, microvessel density

\section{Introduction}

Repeated embryo implantation failure is a form of refractory infertility with unknown etiology (1). It is generally believed that repeated implantation failure is closely related to embryo quality and abnormal endometrial receptivity (2). In vitro fertilization-embryo transfer is the main method for the treatment of infertility. Although some progress has been achieved in clinical practice, some patients still experience repeated implantation failure (3). The key to the success of in vitro fertilization-embryo transfer is endometrial receptivity. Factors affecting endometrial receptivity include endometrial thickness, blood supply and other factors (4-6). Vaginal color Doppler ultrasonography is a non-invasive and easy to operate imaging method with great clinical application in the detection of endometrial receptivity that has attracted more attention lately (7-9). However, the blood flow parameters used in the evaluation of endometrial receptivity by vaginal color Doppler ultrasonography have not been determined (10). This study aimed to investigate the features of uterine artery blood flow by vaginal color Doppler ultrasonography in patients with repeated embryo implantation failure, and to explore the correlation between uterine artery blood flow parameters and microvessel density (MVD) to develop a feasible diagnostic method to predict the outcomes of embryo transfer.

\section{Patients and methods}

Patient information. We recruited 80 patients who underwent in vitro fertilization and embryo transfer therapy in the Third Affiliated Hospital of Zhengzhou University (Henan, China) from September 2014 to January 2016. The ages ranged from 23 to 38 years with a mean age of $32.6 \pm 1.7$ years. The number of basal sinus follicles was 6-10 and the average number of basal ganglia was $8.46 \pm 1.65$. Inclusion criteria: i) The causes of infertility were tubal factors or factors from their male partner, all the patients received in vitro fertilization-embryo transfer, and their age was below 40 years; ii) basal follicle stimulating hormone (bFSH) levels $<10 \mathrm{mIU} / \mathrm{ml}$; iii) more than 8 eggs were obtained per in vitro fertilization-embryo transfer treatment cycle and more than 5 could be implanted; and iv) all the patients or their family members signed the informed consent. Exclusion criteria: i) Hydrosalpinx; ii) endometrial damage; iii) chromosomal abnormalities; iv) endocrine abnormalities; 

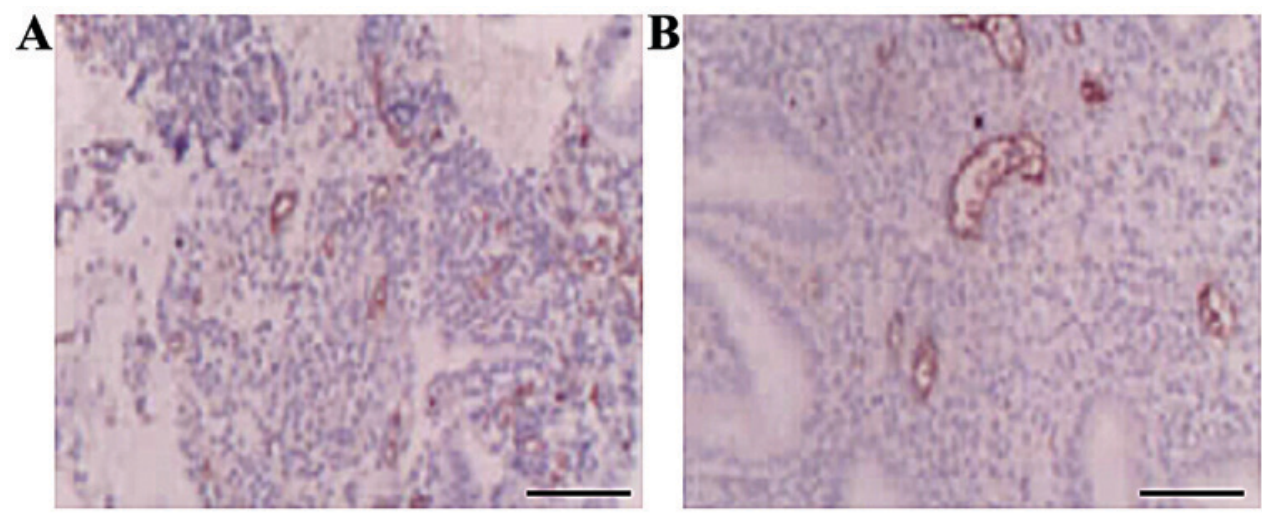

Figure 1. Comparison of MVD between pregnancy group (A) and repeated implantation failure group (B). Scale bar, $200 \mu \mathrm{m}$. MVD, microvessel density.

Table I. Endometrial thickness and uterine artery blood flow parameters.

\begin{tabular}{|c|c|c|c|c|c|c|c|}
\hline \multirow[b]{2}{*}{ Groups } & \multirow{2}{*}{$\begin{array}{l}\text { HCG endometrial } \\
\text { thickness } / \mathrm{mm}\end{array}$} & \multicolumn{3}{|c|}{ Left uterine artery blood flow } & \multicolumn{3}{|c|}{ Right uterine artery blood flow } \\
\hline & & $\mathrm{S} / \mathrm{D}$ & PI & RI & $\mathrm{S} / \mathrm{D}$ & PI & RI \\
\hline $\begin{array}{l}\text { Repeated implantation } \\
\text { failure }(n=30)\end{array}$ & $0.91 \pm 0.26$ & $7.8 \pm 2.4$ & $2.4 \pm 0.4$ & $0.95 \pm 0.12$ & $7.7 \pm 0.6$ & $2.3 \pm 0.3$ & $0.95 \pm 0.12$ \\
\hline Pregnancy $(\mathrm{n}=50)$ & $0.89 \pm 0.24$ & $7.7 \pm 2.1$ & $2.3 \pm 0.3$ & $0.78 \pm 0.11$ & $7.6 \pm 0.5$ & $2.2 \pm 0.2$ & $0.78 \pm 0.11$ \\
\hline t-test & 0.124 & 1.043 & 0.156 & 8.336 & 0.137 & 0.842 & 8.336 \\
\hline P-value & $>0.05$ & $>0.05$ & $>0.05$ & $<0.05$ & $>0.05$ & $>0.05$ & $<0.05$ \\
\hline
\end{tabular}

HCG, human chorionic gonadotropin; PI, pulsatility index; RI, resistance index.

and v) combined with thyroid disease and other autoimmune diseases. A follow-up study was performed on the outcomes of the 80 patients subjected to in vitro fertilization and embryo transfer. We divided the patients into the pregnancy group (successful pregnancy, $n=50$ ) or repeated implantation failure group (more than 2 failures of in vitro fertilization-embryo transfer, $n=30$ ). The study was approved by the Ethics Committee of the Third Affiliated Hospital of Zhengzhou University (Henan, China).

Vaginal color Doppler ultrasonography. The same color ultrasound instrument (Fukuda Denshi Co., Ltd., Tokyo, Japan) was used for this study and all the procedures were performed by the same ultrasound physician. The vaginal ultrasound probe $(8 \mathrm{MHz})$ was used in all the subjects. Patients received human chorionic gonadotropin (HCG). We detected the following endometrium blood parameters by ultrasound: Endometrial thickness, peak systolic velocity/end diastolic velocity (S/D), pulsatility index (PI) and resistance index (RI).

SP method to detect F8 factor-labeled microvessels. On day 10 of the menstrual cycle, the test was carried out by using the urine testosterone (LH) test paper. On day 8 after the urine LH peak, the uterine endometrial tissue was scraped and collected using a curette (no. 5) after PI test. The endometrial tissue was fixed in $10 \%$ formaldehyde solution and embedded in paraffin. The endometrial tissue specimens were then subjected to the
Table II. Correlation analysis of uterine artery blood flow parameters (S/D, PI and RI) and MVD.

\begin{tabular}{lcc}
\hline Indexes & r value & P-value \\
\hline S/D & 0.082 & $>0.05$ \\
PI & 0.176 & $>0.05$ \\
RI & 0.515 & $<0.05$ \\
\hline
\end{tabular}

PI, pulsatility index; RI, resistance index; MVD, microvessel density.

SP method. Primary rabbit anti-human F8 factor polyclonal antibody and anti-rabbit secondary antibody were both purchased from Shanghai Qunji Biotech Co., Ltd. (Shanghai, China). All procedures were performed according to the manufacturer's instructions. Primary antibody was replaced with phosphate-buffered saline (PBS) to serve as a negative control, and F8 factor-positive tissue was used as a positive control.

Indicator observation and the result determination. i) The endometrial thickness and uterine artery blood flow parameters (S/D, PI and RI) were measured. ii) MVD counting: The vascular endothelial cell marker F8 factor-associated antigen was stained in specimens. Tissue sections were observed under the microscope and the mean number of MVDs of 5 visual fields were recorded. The stained endothelial cells 
were counted according to 1 MVD (11). iii) Investigation of the correlation between uterine artery blood flow parameters (S/D, PI and RI) and MVD in the repeated implantation failure group.

Statistical analysis. Statistical analysis was performed using SPSS 20.0 statistical software (IBM SPSS, Armonk, NY, USA). The measurement data were expressed as mean \pm standard deviation (mean \pm SD) and analyzed by t-test. The measurement data were analyzed by $\chi^{2}$ test. The correlation analysis was performed using Spearman correlation analysis. $\mathrm{P}<0.05$ was considered to indicate a statistically significant difference.

\section{Results}

Endometrial thickness and uterine artery blood flow. We first examined the endometrial thickness and uterine blood flow in the pregnancy and repeated implantation failure groups. As shown in Table I, we found no significant differences in the thickness of endometrium (HCG endometrial thickness) and uterine artery blood flow parameters (S/D and PI) between the two groups ( $p>0.05)$. The RI value of endometrial blood flow in the repeated implantation failure group was significantly higher than that in the pregnancy group $(\mathrm{p}<0.05)$.

Endometrial MVD. The MVD in the repeated implantation failure group was $3.46 \pm 0.84$, which was significantly lower than that the $5.52 \pm 1.03$ of the pregnancy group $(t=9.027$, $\mathrm{p}<0.05$ ) (Fig. 1). Immunohistochemistry of vascular endothelial cell marker F8 factor showed that MVD was significantly higher in the pregnancy group than that in repeated implantation failure group.

Correlation between uterine artery blood flow parameters and MVD. Spearman correlation analysis showed that the S/D value of uterine artery blood flow in the repeated implantation failure group was not correlated with MVD $(r=0.082, \mathrm{p}>0.05)$ or uterine artery blood flow PI $(r=0.176, p>0.05)$, but was positively correlated with RI $(\mathrm{r}=0.515, \mathrm{p}<0.05)$ (Table II).

\section{Discussion}

The clinical application of in vitro fertilization-embryo transfer technology has attracted recent attention. Although this treatment increases the rate of pregnancy, the successful rate of single embryo implantation is still unsatisfactory (12). Around $30 \%$ of embryos are successfully implanted under natural conception conditions, but the success rate of individual embryos during in vitro fertilization and embryo transfer is only $10-15 \%(13,14)$. Factors affecting the effectiveness of in vitro fertilization and embryo transfer therapy are endometrial receptivity and embryonic factors.

Studies have shown that vaginal color Doppler ultrasound can predict and evaluate endometrial receptivity through the thickness and shape of the endometrium (15-17). Some scholars believe that endometrial blood flow is closely related with endometrial receptivity and is beneficial for embryo implantation and development $(18,19)$. Studies have confirmed that the higher MVD value usually leads to more abundant capillaries in newborns (20). Therefore, endometrial MVD during the implantation window can predict endometrial receptivity. F8 factor is an important vascular endothelium-specific marker that is often used as a marker for MVD (21). Our results show that endometrium MVD in the repeated implantation failure group was significantly lower than that of the MVD in the pregnancy group. Thus, endometrial angiogenesis is more robust in patients with successful pregnancy, which in turn promotes the formation of new blood vessels that benefit embryo implantation, and increase the success rate of pregnancy, which is consistent with previous studies (22).

Further analysis showed no significant differences in the endometrial thickness (HCG endometrial thickness) and uterine artery blood flow (S/D and PI) between the two groups. The RI value of endometrial blood flow in repeated implantation group was significantly higher than that in pregnancy group. Spearman correlation analysis showed that the S/D value of uterine artery blood flow in repeated implantation failure group was not correlated with MVD and uterine artery blood flow PI, but was positively correlated with RI. Thus, MVD in neonatal blood vessels can be assessed by detecting the endometrial blood flow RI values using color Doppler ultrasonography, which provides a simple method for clinical detection and determination of endometrial angiogenesis.

In conclusion, endometrium MVD in patients with repeated implantation failure during the implantation window was significantly lower than that in patients with successful in vitro fertilization and embryo transfer. We found a significant difference in uterine artery blood flow RI between the two groups. The uterine artery blood flow RI value in patients with repeated implantation failure was positively correlated with MVD, which has clinical significance in predicting the outcome of embryo transfer.

\section{References}

1. Mangla M and Singla D: Transvaginal colour doppler ultrasound in predicting response to chemoradiation in patients with carcinoma of the cervix. S Afr J Gynaecol Oncol 7: 68-72, 2015.

2. El-Danasouri I, Sterzik K, Rinaldi L, Pacchiarotti A, DeSanto M and Selman H: Effect of transferring a morphologically impaired embryo with a good quality embryo on the pregnancy and implantation rates. Eur Rev Med Pharmacol Sci 20: 394-398, 2016.

3. Kim A, Han JE, Yoon TK, Lyu SW, Seok HH and Won HJ: Relationship between endometrial and subendometrial blood flow measured by three-dimensional power Doppler ultrasound and pregnancy after intrauterine insemination. Fertil Steril 94: 747-752, 2010.

4. Hutchison SK, Stepto NK, Harrison CL, Moran LJ, Strauss BJ and Teede HJ: Effects of exercise on insulin resistance and body composition in overweight and obese women with and without polycystic ovary syndrome. J Clin Endocrinol Metab 96: E48-E56, 2011.

5. Erbil Y, Ozluk Y, Giriş M, Salmaslioglu A, Issever H, Barbaros U, Kapran Y, Ozarmağan S and Tezelman S: Effect of lugol solution on thyroid gland blood flow and microvessel density in the patients with Graves' disease. J Clin Endocrinol Metab 92: 2182-2189, 2007

6. Guo TH, Tong XH, Luo LH, Luan HB, Zhou GX and Wan YY: Value of microsurgical varicocelectomy for severe oligo-asthenospermia patients failed in fertilization assisted by in vitro fertilization. Eur Rev Med Pharmacol Sci 20: 1669-1674, 2016.

7. Legro RS, Brzyski RG, Diamond MP, Coutifaris C, Schlaff WD, Casson P, Christman GM, Huang H, Yan Q, Alvero R, et al; NICHD Reproductive Medicine Network: Letrozole versus clomiphene for infertility in the polycystic ovary syndrome. $\mathrm{N}$ Engl J Med 371: 119-129, 2014. 
8. Yang TF, Wang JD, Luo HJ, Wang XY and Li FH: Relationship between ultrasonographic velocimetric parameters and microvessel density in patients with papillary thyroid carcinoma and its clinical significance. Zhonghua Er Bi Yan Hou Tou Jing Wai Ke Za Zhi 42: 126-129, 2007 (In Chinese).

9. Dogan O, Yildiz A, Temizkan O and Pulatoglu C: Comparison of uterine, endometrial and ovarian blood flow by transvaginal color Doppler ultrasound in ovulatory and anovulatory cycles. Ginekol Pol 87: 581-584, 2016.

10. Vizza L, Smith CA, Swaraj S, Agho K and Cheema BS: The feasibility of progressive resistance training in women with polycystic ovary syndrome: A pilot randomized controlled trial BMC Sports Sci Med Rehabil 8: 14, 2016.

11. Weidner N, Folkman J, Pozza F, Bevilacqua P, Allred EN Moore DH, Meli S and Gasparini G: Tumor angiogenesis: A new significant and independent prognostic indicator in early-stage breast carcinoma. J Natl Cancer Inst 84: 1875-1887, 1992.

12. Gu J, Han $\mathrm{CH}, \mathrm{Hu}$ FF, Wang YB and Cao YJ: The correlation analysis of human embryonic MMP-9 secretion and embryo quality. Eur Rev Med Pharmacol Sci 19: 2354-2358, 2015.

13. Salzillo PL, Salzillo ME, Iannella I, Cobellis L and Colacurci N: Sonographic aspects in the study of endometrial receptivity in women undergoing in vitro fertilization. Minerva Ginecol 62: 267-275, 2010 (In Italian).

14. Achache H, Tsafrir A, Prus D, Reich R and Revel A: Defective endometrial prostaglandin synthesis identified in patients with repeated implantation failure undergoing in vitro fertilization. Fertil Steril 94: 1271-1278, 2010.

15. Harapan H and Yeni CM: The role of microRNAs on angiogenesis and vascular pressure in preeclampsia: The evidence from systematic review. Egypt J Med Hum Genet 16: 313-325, 2015.
16. Uysal S, Ozbay EP, Ekinci T, Aksüt H, Karasu S, Isık AZ and Soylu F: Endometrial spiral artery Doppler parameters in unexplained infertility patients: Is endometrial perfusion an important factor in the etiopathogenesis? J Turk Ger Gynecol Assoc 13: 169-171, 2012.

17. Gibson DA, Greaves E, Critchley HO and Saunders PT: Estrogen-dependent regulation of human uterine natural killer cells promotes vascular remodelling via secretion of CCL2. Hum Reprod 30: 1290-1301, 2015.

18. Coughlan C, Ledger W, Wang Q, Liu F, Demirol A, Gurgan T, Cutting R, Ong K, Sallam H and Li TC: Recurrent implantation failure: Definition and management. Reprod Biomed Online 28: 14-38, 2014.

19. Fatemi HM and Popovic-Todorovic B: Implantation in assisted reproduction: A look at endometrial receptivity. Reprod Biomed Online 27: 530-538, 2013.

20. Dechaud H, Bessueille E, Bousquet PJ, Reyftmann L, Hamamah S and Hedon B: Optimal timing of ultrasonographic and Doppler evaluation of uterine receptivity to implantation. Reprod Biomed Online 16: 368-375, 2008

21. Anicic R, Djukic M, Rakic S, Vasiljevic M, Dimitrijevic D and Milicevic S: Evaluation of utero-ovarian hemodynamics in relation to fertility and stage of endometriosis. Clin Exp Obstet Gynecol 39: 526-528, 2012

22. Ceyhan L, Cagri Savas M, Baspinar S, Duman L and Büyükyavuz BI: The correlation between preputial blood flow and microvessel density in distal hypospadias: A prospective clinical study. J Pediatr Urol 10: 103-106, 2014. 